This is a peer-reviewed, accepted author manuscript of the following commentary Garbin, B., Fatome, J., Oppo, G-L., Erkintalo, M., Murdoch, S. G., \& Coen, S. (2021). Dissipative polarization domain walls in a passive coherently driven Kerr resonator. Physical Review Letters, 126, [023904]. https://doi.org/10.1103/PhysRevLett.126.023904

\title{
Dissipative polarization domain walls in a passive coherently-driven Kerr resonator
}

\author{
Bruno Garbin,,${ }^{1,2,3}$ Julien Fatome, ${ }^{1,2,4}$ Gian-Luca Oppo, ${ }^{5}$ \\ Miro Erkintalo, ${ }^{1,2}$ Stuart G. Murdoch, ${ }^{1,2}$ and Stéphane Coen ${ }^{1,2, *}$ \\ ${ }^{1}$ Physics Department, The University of Auckland, \\ Private Bag 92019, Auckland 1142, New Zealand \\ ${ }^{2}$ The Dodd-Walls Centre for Photonic and Quantum Technologies, Dunedin, New Zealand \\ ${ }^{3}$ Université Paris-Saclay, CNRS, Centre de Nanosciences et de Nanotechnologies, 91120, Palaiseau, France \\ ${ }^{4}$ Laboratoire Interdisciplinaire Carnot de Bourgogne (ICB), \\ UMR 6303 CNRS, Université de Bourgogne Franche-Comté, \\ 9 Avenue Alain Savary, BP 47870, F-21078 Dijon, France \\ ${ }^{5}$ SUPA and Department of Physics, University of Strathclyde, Glasgow G4 ONG, Scotland, European Union
}

\begin{abstract}
Using a passive, coherently-driven nonlinear optical fiber ring resonator, we report the experimental realization of dissipative polarization domain walls. The domain walls arise through a symmetry breaking bifurcation and consist of temporally localized structures where the amplitudes of the two polarization modes of the resonator interchange, segregating domains of orthogonal polarization states. We show that dissipative polarization domain walls can persist in the resonator without changing shape. We also demonstrate on-demand excitation, as well as pinning of domain walls at specific positions for arbitrary long times. Our results could prove useful for the analog simulation of ubiquitous domain-wall related phenomena, and pave the way to an all-optical buffer adapted to the transmission of topological bits.
\end{abstract}

Domain walls (DWs) are self-localized kink-type topological defects that connect two stable states of a physical system. They usually form through a spontaneous symmetry breaking bifurcation [1], and are found in various contexts, including magnetism [2], hydrodynamics [3], biology [4], Bose-Einstein condensates [5], and string theory [6]. The paradigmatic examples are the interfaces that separate domains with distinct magnetization in ferromagnetic materials $[2,7]$, whose unique properties are exploited in modern spintronics devices to store or even transfer information [8]. Additionally, DWs are central to numerous phase transitions in condensed matter and quantum physics $[4,9]$.

DWs are also known to manifest themselves in optical systems. In this context, the terminology was first used to describe stationary spatial distributions of light arising from the pure nonlinear (Kerr) interactions of counter-propagating beams [10] (and reported experimentally in [11]). Subsequently, Haelterman and Sheppard introduced the concept of DW solitons by describing vector, propagating structures, segregating homogeneous domains of orthogonal polarization states, and that resist diffractive (transverse) or dispersive (temporal) spreading in Kerr media [12]. Referblack to as polarization DWs (PDWs), these structures have only recently been convincingly observed experimentally - more than two decades after their theoretical description - in the single-pass, conservative, propagation configuration of a normally dispersive single-mode optical fiber [13]. Remarkably, this experiment has demonstrated the potential of temporal PDWs for transmission of topological bits, robust to noise and nonlinear impairments, as originally foreseen [14].

Here we experimentally demonstrate dissipative op- tical PDW solitons. These structures are realized in a coherently-driven passive Kerr ring resonator, where they can circulate indefinitely. Their existence relies on a spontaneous symmetry breaking (SSB) instability of the intracavity field [15], which constitutes a fundamental difference with respect to the conservative PDWs of Refs. [12, 13]. Our dissipative PDWs enable all-optical storage of temporal PDWs and constitute a key technology in supporting potential topologically-robust transmissions. Dissipative PDWs could also pave the way to real-time, stochastic, room temperature analog simulations of DW-related solid-state physics phenomena not easily observable in other settings [16-18]. Additionally, by analogy with phase DWs pblackicted in the transverse structure of optical parametric [19] and four-wave mixing [20] oscillators, which are related to the Ising Hamiltonian [21, 22], domains of orthogonal polarizations segregated by dissipative PDWs could be associated with different spin states and provide a new route to solve complex optimization problems. We note that earlier observations in fiber lasers may constitute signatures of chaotic ensembles of many PDWs [23-25]. The results of Marino et al [26] can also be interpreted in terms of dissipative PDW-like structures, but these were obtained in a hybrid electro-optical system. In contrast, the results we present in this Letter provide the first evidence of isolated dissipative all-optical temporal PDWs.

We start by describing theoretically how dissipative PDWs emerge in our system. We consider a dispersive passive ring resonator that is externally and coherently driven by a continuous-wave (cw) light beam and that exhibits a Kerr nonlinearity. The intracavity field is described in terms of the complex amplitudes, $E_{1,2}$, of the two orthogonal polarization modes of the resonator. In 
the mean-field limit, the temporal evolution of these two modal amplitudes can be described by normalized coupled Lugiato-Lefever equations [27-30],

$$
\begin{aligned}
\frac{\partial E_{1,2}}{\partial t}=\left[-1+i\left(\left|E_{1,2}\right|^{2}\right.\right. & \left.+B\left|E_{2,1}\right|^{2}-\Delta\right) \\
& \left.-i \frac{\partial^{2}}{\partial \tau^{2}}\right] E_{1,2}+\sqrt{X / 2} .
\end{aligned}
$$

The terms on the right hand side describe respectively cavity losses, self- and cross-phase modulation, the cavity phase detuning, chromatic dispersion (taken as normal, to avoid modulational instabilities of $\mathrm{cw}$ stationary states of the resonator [28]), and external driving. We consider here identical detuning and driving strength for the two modes. $t$ represents a slow time over which the evolution of the intracavity field takes place, at the scale of the cavity photon lifetime, while $\tau$ is a fast-time that describes the temporal structure of the field along the resonator round trip. $B$ is the cross-phase modulation coefficient. In optical fibers (as used in our experiments), $B$ can be as large as 2 for circularly polarized modes [31], but PDW existence only requires $B>1[12]$. Finally, $\Delta$ is the detuning parameter, which measures the separation between the driving laser frequency and the nearest cavity resonance in terms of the cavity half-linewidth, and $X$ is the normalized total driving power.

Representative stationary $(\partial / \partial t=0) \mathrm{cw}(\partial / \partial \tau=0)$ solutions of the above equations are illustrated in Fig. 1(a). Because the equations are symmetric with respect to an interchange of the two polarization modes, $E_{1} \rightleftarrows E_{2}$, the simplest stationary solutions express this symmetry $\left(E_{1}=E_{2}\right)$. Here we retrieve the characteristics S-shaped, bistable response of the Kerr cavity [yellow curve in Fig. 1(a)]. However, above a certain threshold of driving power, the upper-state solution undergoes SSB: the intensity of the two polarization modes part (blue and orange curves) [28, 32, 33]. Because of the symmetry of the system, there exists two such solutions, mirror-images of each other, in which a different mode dominates. These solutions correspond to intracavity fields that are overall elliptically polarized, with opposite handedness. This polarization SSB has been recently observed experimentally [34]. When the two symmetry-broken solutions are simultaneously excited in different regions (or domains) of the resonator, there exists a shape-preserving temporal structure interconnecting them and across which the two polarization modes interchange: the PDW [Fig. 1(b)]. Note how the total intensity (black curve) is nearly constant across the PDW. As shown numerically in [15], these dissipative PDWs can circulate indefinitely around the driven resonator without losing power or changing shape. Their robustness stems from a double balance, similar to that realized for temporal cavity solitons [35]: external driving compensates losses, while dispersive spreading is balanced by nonlin-

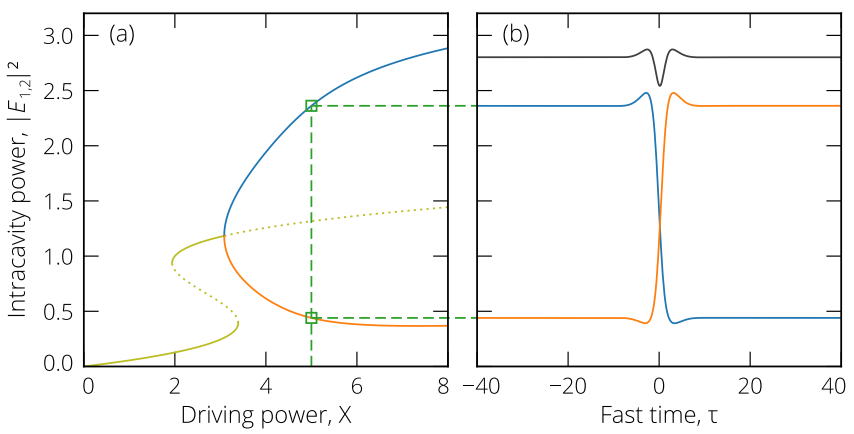

FIG. 1. Numerical illustration of the polarization SSB and associated PDWs of Eqs. (1) for $\Delta=3$ and $B=2$. (a) Stationary cw intracavity intensities versus driving power $X$. Yellow curve: symmetric solutions $\left(E_{1}=E_{2}\right.$; dotted lines are unstable states); blue and orange curves: asymmetric solutions $\left(E_{1} \neq E_{2}\right)$. (b) Temporal intensity profile of a single PDW connecting the two cw symmetry-broken solutions existing for $X=5$. Blue and orange curves correspond to the two polarization modes, while the black curve shows the total power.

earity. In contrast to the single pass configuration, this double balance uniquely fixes the duration and power levels of dissipative PDWs.

The experimental setup that we have used to realize and control dissipative PDWs is depicted in Fig. 2. It is based on $\mathrm{a} \simeq 10 \mathrm{~m}$-long passive optical fiber ring resonator mostly built out of highly nonlinear, normal dispersion, "spun" fiber, exhibiting very low birefringence due to twisting applied at the drawing stage [36]. The ring is closed with two SMF-28 fiber couplers, with splitting ratio $90 / 10$ and 99/1, enabling injection of the driving and monitoring of the intracavity field, respectively. Overall the resonator exhibits normal dispersion at the 1550-nm driving wavelength, with averaged second order dispersion coefficient $\left\langle\beta_{2}\right\rangle \simeq 53 \mathrm{ps}^{2} / \mathrm{km}$ and nonlinear coefficient $\langle\gamma\rangle \simeq 4.3 \mathrm{~W}^{-1} \mathrm{~km}^{-1}$. The free-spectral-range is found to be $19.8 \mathrm{MHz}$, corresponding to a round-trip time $t_{\mathrm{R}}$ of $50.6 \mathrm{~ns}$. The measublack finesse is about 24, amounting to losses of $26 \%$ per round-trip, a photon lifetime of about $4 t_{\mathrm{R}}$, and a resonance width of $825 \mathrm{kHz}$.

The resonator is synchronously driven with flat-top 1.1 ns pulses [37]. These pulses are obtained by carving the cw output of a $1 \mathrm{kHz}$ linewidth, erbiumdoped distributed-feedback fiber laser with a $10 \mathrm{GHz}-$ bandwidth Mach-Zehnder amplitude modulator (AM). The AM is followed by a fast polarization modulator (PM) used to apply perturbations to the driving polarization as explained below. The two modulators are connected to separate pattern generators (PG) synchronized to the same $\simeq 10 \mathrm{GHz}$ sinusoidal clock, set at a harmonic of the FSR. Before injection into the resonator, the driving pulses are amplified up to $15 \mathrm{~W}$ peak power (corresponding to $X$ values up to 30 ) using an erbium-doped fiber amplifier (EDFA) combined with a band-pass filter $(\mathrm{BPF})$ for rejection of amplified spontaneous emission 


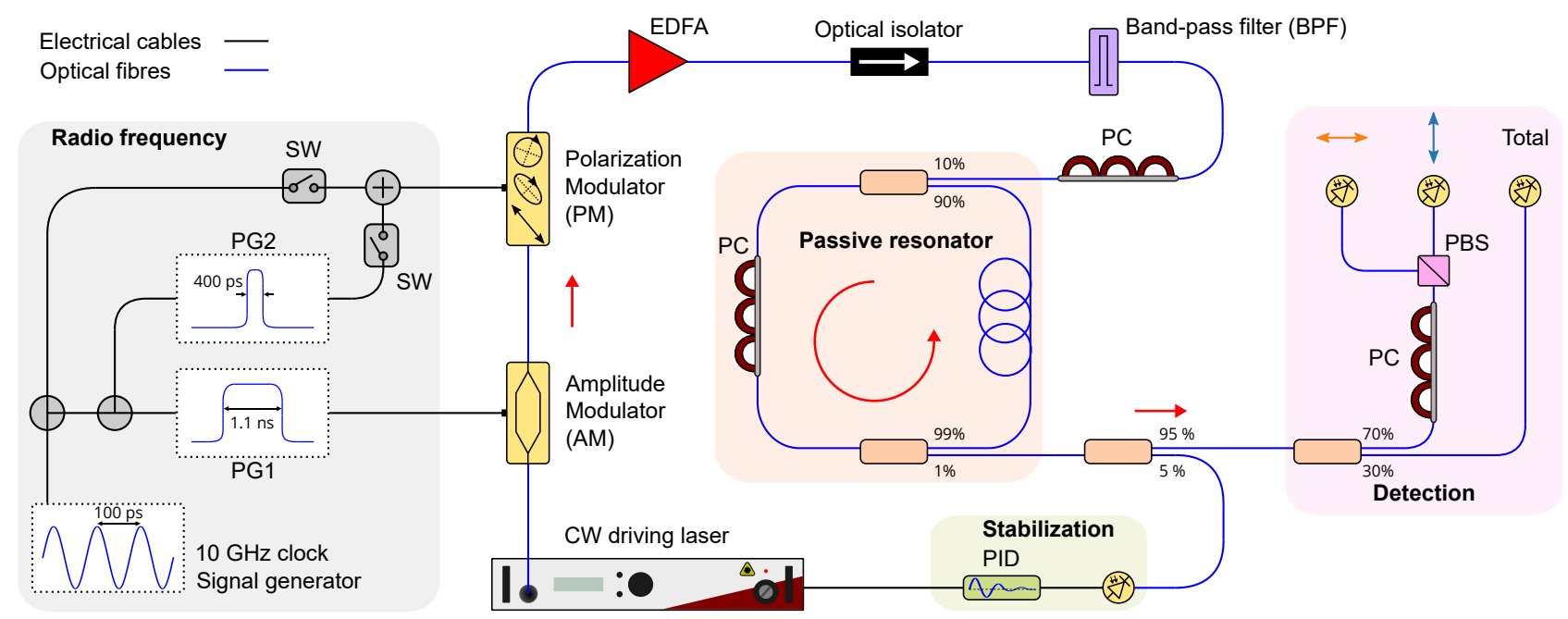

FIG. 2. Experimental set-up. The passive fiber ring resonator is highlighted with an orange background. PG1 and PG2, pattern generators; SW, electrical switch; EDFA, erbium-doped fibre amplifier; PC, polarization controller; PBS, polarizing beamsplitter.

noise. At the output, we monitor separately the power of the two polarization modes, split by a polarizing beamsplitter (PBS) preceded by a polarization controller (PC), as well as the total output power. These three signals are measublack with a triplet of $12.5 \mathrm{GHz}$-bandwidth amplified photodiodes. Additionally, a small fraction of the total output power is monitoblack and maintained constant by a PID feedback controller acting on the driving laser frequency, for stabilization of the detuning with respect to environmental fluctuations.

PDWs require interchange symmetry between the two polarization modes of the resonator. Our optical fiber ring is however slightly birefringent, due to the couplers, which are not built out of spun fiber, as well as unavoidable fiber bending. To counterbalance the residual cavity birefringence, a PC is incorporated into the fiber ring. In this configuration, the polarization modes are associated with states of polarization that evolve around the fiber ring, and that map onto themselves over one round-trip [38]. This evolution is averaged in the mean-field model, Eqs. (1) [30]. Another PC, inserted before the input coupler, is used to project the driving field equally onto the two modes, and realize balanced driving conditions.

In practice, the setup is adjusted by observing the resonances of the two polarization modes while scanning the driving laser frequency. A position of the intracavity PC is found for which, close to a point where the resonances overlap, their separation can be tuned without affecting their relative amplitudes. With the two resonances slightly apart, i) the output PC is set to correctly separate the modes in the detection stage, and ii) driving is balanced by matching the amplitudes of the observed resonances. Birefringence is then cancelled by superimposing the two resonances. Finally, we increase the driving power until we observe the polarization SSB described in Fig. 1(a) (and reported in [34]), and we lock the detuning within the region where SSB occurs.

To proceed with observations of PDWs, we record the output power levels across our driving pulses over subsequent cavity round-trips using a 13-GHz-bandwidth realtime oscilloscope. A typical evolution is shown as color plots (bottom-to-top) in Figs. 3(a)-(c), with the three panels corresponding respectively to the powers of the two separate polarization modes and their total. Using matching colors, line plots are also presented in Fig. 3(d) for selected round trips. We start in a symmetry-broken state, where the "orange" mode uniformly dominates; see round trip \#500 in (d). After about 1000 cavity roundtrips, a localized, 400 ps-wide, rf perturbation is applied for about 20 round-trips to the PM. This carves a domain of different polarization in the middle of the driving pulse. We then let the intracavity field evolve freely for the rest of the measurement. Shortly after applying the perturbation, we observe at the output a sudden increase of the "blue mode" at a location corresponding to the perturbation, correlated with a depression of the "orange mode"; see round trip \#1100 in (d). We now have a "domain" where the blue mode dominates embedded within the original orange-dominated state. In that domain, the power levels of the two polarization modes have essentially been interchanged, reflecting the mirror symmetry of the system. This symmetry can be further appreciated by noting that the color plots of the two polarization components measublack throughout the experiment [Figs. 3(a) and (b)] are essentially negatives of each other. Correspondingly, the total output power [Fig. 3(c) and black curves in (d)] reveals little sign of the polarization structure of the intracavity field. We clearly are in presence of an almost pure polarization dynamics.

We identify the transition regions, along the fast- 

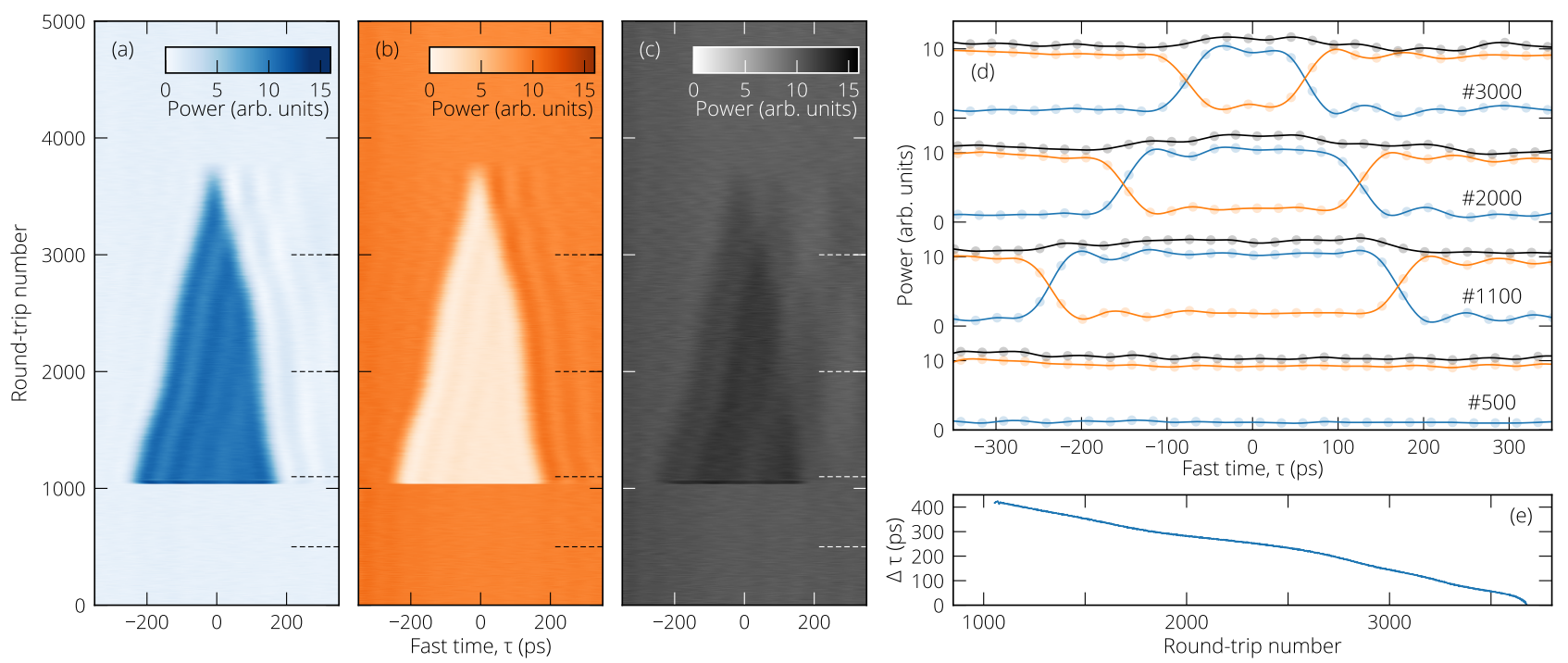

FIG. 3. Experimental evidence of dissipative PDWs for $X \simeq 30.5$ and $\Delta \simeq 13.5$. (a)-(c) Round-trip-by-round-trip evolution of the fast-time output power profile for the two polarization components, $\left|E_{1,2}(\tau)\right|^{2}[(\mathrm{a})$, blue, and (b), orange] as well as for the total signal [(c), grey] before and after a short, localized, 400 ps-long polarization perturbation is applied at about round-trip \#1000. The perturbation generates a "blue"-mode dominated domain connected to the surrounding regions by two PDWs. The PDWs drift towards each other, eventually mutually annihilating. (d) provides corresponding line plots using matching colors for selected round-trips (marked with dashed side-lines in (a)-(c)]. (e) Evolution of the temporal separation $\Delta \tau$ between the PDWs.

time $(\tau)$ coordinate, where the field switches polarization as two PDWs of opposite symmetry. Figure 3 reveals that these PDWs slowly drift towards each other (at a rate of about $0.15 \mathrm{ps} / t_{\mathrm{R}}$ ); see also panel (e) where we plot the temporal separation between the PDWs vs round-trip number. This results in the shrinkage of the blue-mode dominated domain created by the polarization perturbation. The PDWs eventually collide and mutually annihilate (around round trip \#3800), reverting the system to its initial state. If the interchange symmetry between the polarization modes was perfect, the PDWs would have no preferblack direction of motion and would remain still. We can therefore attribute the PDWs' motion to the presence of residual asymmetries, favoring one state over the other [34]. In particular, from the slight excess power visible in the central domain [Fig. 3(c)], we can infer that the blue mode may have been driven slightly stronger than the orange mode. Numerical simulations made by introducing such asymmetry into Eqs. (1) confirm this interpretation. Nevertheless, the PDWs are very robust: they persist for nearly one thousand photon lifetimes while maintaining their shape (as far as the $80 \mathrm{ps}$ temporal resolution of our real-time oscilloscope allows us to judge), demonstrating their dissipative and nonlinearly-localized character.

To observe stationary PDWs, we have investigated the use of an external modulation of parameters to trap PDWs, as that technique has been successfully exploited to pin various types of moving fronts in other nonlinear systems $[26,39-42]$. In our case, we modulate the polar- ization of the driving field, by applying a small fraction of the $10 \mathrm{GHz}$ sinusoidal clock signal to the PM (see Fig. 2). This modulation can be turned on and off with an additional rf switch (SW). Figure 4(a) shows the result of an experiment that starts like that discussed in Fig. 3 (only showing one polarization component), with two PDWs initially drifting towards each other at a constant speed. When the modulation is turned on at roundtrip \#1200 [see Fig. 4(b) as well as the black-shaded area in Fig. 4(a)], we immediately observe a change of behavior. The PDWs visibly change their drift velocities, and after some transient, reach a fixed position with respect to the modulation. In that position, the PDW drift imparted by the local driving imbalance associated with the modulation counteracts the original motion due to the residual asymmetries. The PDWs hold their position until the modulation is turned off at roundtrip \#2500, which releases them, back on their original collision course. In Fig. 4(c), using the same technique, we demonstrate long term pinning of two PDWs over 30 seconds (corresponding to a propagation distance of $6 \times 10^{6} \mathrm{~km}$ inside the resonator), which has enabled us to measure their temporal intensity profile with a faster, 65-GHz, sampling oscilloscope [blue dots in Fig. 4(d)]. This measurement demonstrates that our PDWs have a rise time (10-90\%) of less than $9 \mathrm{ps}$, limited by the bandwidth of the sampling oscilloscope, and compatible with the $1.6 \mathrm{ps}$ numerical expectation [green curves in Fig. 4(d)].

We remark that the PDWs reported in Figs. 3 and 4 

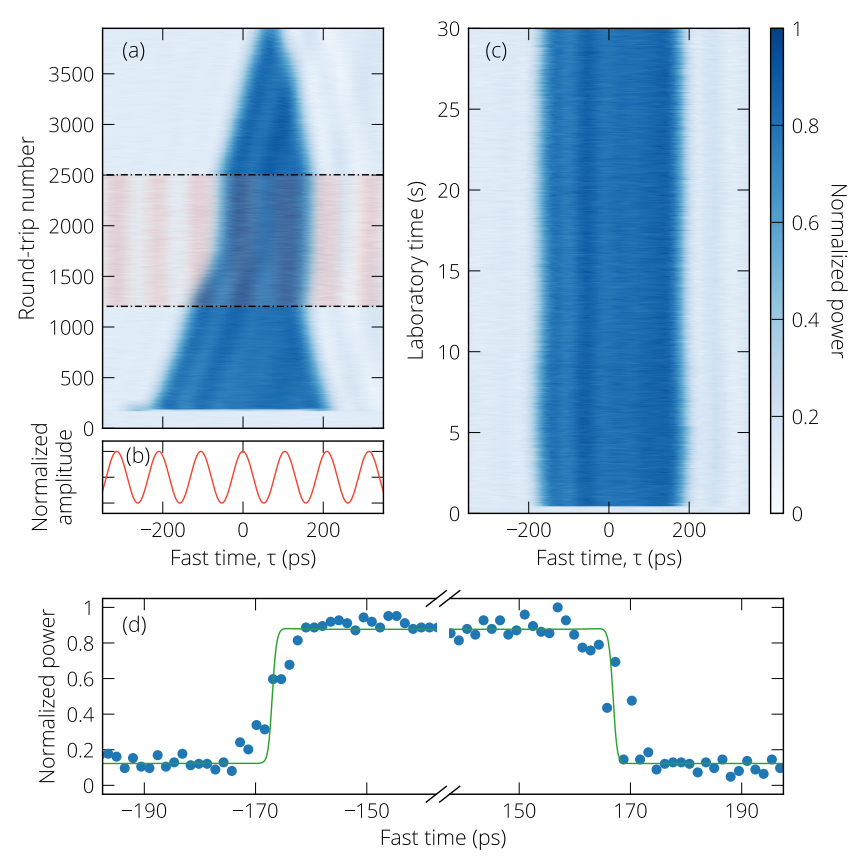

FIG. 4. (a) Demonstration of drifting PDWs being pinned/unpinned to a shallow modulation of the driving polarization. We only show the evolution of the output power of one polarization component. The $10 \mathrm{GHz}$ sinusoidal modulation [transparent shades of black and panel (b)] is applied between round-trips \#1200 and 2500. (c) Long term pinning of PDWs, for $X \simeq 24$ and $\Delta \simeq 9.2$. (d) Sampling scope measurement (blue dots) of the temporal intensity profile of the two PDWs trapped in (c). The green curve is the numerical expectation.

are observed for driving powers comparatively larger than that consideblack in the theoretical plot of Fig. 1. Large driving powers are made necessary by the presence of a small amount of linear coupling between the polarization modes of our fiber resonator. Numerical calculations indicate that linear coupling, which splits the cavity resonance [43], thwarts polarization SSB at low power. SSB and PDWs are restoblack at high power, when the Kerrinduced tilt of the cavity resonance dominates over the splitting [44].

In conclusion, we have reported here the first experimental demonstration of isolated optical dissipative PDWs. The PDWs are recirculated in a passive, coherently-driven Kerr optical fiber ring resonator. Their existence relies on a symmetry breaking bifurcation and on an interchange symmetry between the two polarization modes of the resonator. Our dissipative PDWs are found to be robust with respect to residual imperfections and asymmetries, and can be pinned to a shallow external modulation. Given their duration, our resonator could hold up to 20,000 PDWs in a cw-driven configuration, which could be achieved by mitigating linear mode coupling as in [34], thus blackucing power requirements. Our results suggest that our system could be used as an all-optical buffer for PDW-based topological bit transmissions [13]. Optical PDWs could also prove useful for the real time stochastic analog simulation of other DWrelated phenomena.

We thank Y. Wang for technical help, and F. Leo for fruitful discussions. We acknowledge financial support from The Royal Society of New Zealand, in the form of Marsden Funding (18-UOA-310), as well as James Cook (JCF-UOA1701, for S.C.) and Rutherford Discovery (RDF-15-UOA-015, for M.E.) Fellowships. J.F. thanks the Conseil régional de Bourgogne Franche-Comté, mobility (2019-7-10614).

* s.coen@auckland.ac.nz

[1] M. Golubitsky, I. Stewart, and D. G. Schaeffer, Singularities and Groups in Bifurcation Theory: Volume II, Applied Mathematical Sciences No. 69 (Springer-Verlag, New York, 1988).

[2] P.-E. Weiss, "L'hypothèse du champ moléculaire et la propriété ferromagnétique," J. Phys. Theor. Appl. 6, 661 (1907).

[3] F. Tsitoura, U. Gietz, A. Chabchoub, and N. Hoffmann, "Phase domain walls in weakly nonlinear deep water surface gravity waves," Phys. Rev. Lett. 120, 224102 (2018).

[4] L. E. Reichl, A Modern Course in Statistical Physics, 4th ed. (Wiley-VCH, 2016).

[5] S. Coen and M. Haelterman, "Domain wall solitons in binary mixtures of Bose-Einstein condensates," Phys. Rev. Lett. 87, 140401 (2001); D. M. Stamper-Kurn and M. Ueda, "Spinor Bose gases: Symmetries, magnetism, and quantum dynamics," Rev. Mod. Phys. 85, 1191 (2013).

[6] S. Weinberg, The Quantum Theory of Fields, Vol. 2 (Cambridge University Press, 1995).

[7] D. Y. Parpia, B. K. Tanner, and D. G. Lord, "Direct optical observation of ferromagnetic domains," Nature 303, 684 (1983); J. Unguris, R. J. Celotta, and D. T. Pierce, "Observation of two different oscillation periods in the exchange coupling of $\mathrm{Fe} / \mathrm{Cr} / \mathrm{Fe}(100)$," Phys. Rev. Lett. 67, 140 (1991).

[8] D. A. Allwood, G. Xiong, C. C. Faulkner, D. Atkinson, D. Petit, and R. P. Cowburn, "Magnetic domainwall logic," Science 309, 1688 (2005); S. S. P. Parkin, M. Hayashi, and L. Thomas, "Magnetic domainwall racetrack memory," ibid. 320, 190 (2008); J. A. Currivan-Incorvia, S. Siddiqui, S. Dutta, E. R. Evarts, J. Zhang, D. Bono, C. A. Ross, and M. A. Baldo, "Logic circuit prototypes for three-terminal magnetic tunnel junctions with mobile domain walls," Nat. Commun. 7, 10275 (2016).

[9] A. Dutta, G. Aeppli, B. K. Chakrabarti, U. Divakaran, T. F. Rosenbaum, and D. Sen, Quantum Phase Transitions in Transverse Field Spin Models: From Statistical Physics to Quantum Information (Cambridge University Press, Cambridge, 2015).

[10] V. E. Zakharov and A. V. Mikhailov, "Polarization domains in nonlinear optics," JETP Lett. 45, 349 (1987).

[11] S. Pitois, G. Millot, and S. Wabnitz, "Polarization domain wall solitons with counterpropagating laser beams," 
Phys. Rev. Lett. 81, 1409 (1998).

[12] M. Haelterman and A. P. Sheppard, "Polarization domain walls in diffractive or dispersive Kerr media," Opt. Lett. 19, 96 (1994); A. P. Sheppard and M. Haelterman, "Polarization-domain solitary waves of circular symmetry in Kerr media," ibid. 19, 859 (1994).

[13] M. Gilles, P.-Y. Bony, J. Garnier, A. Picozzi, M. Guasoni, and J. Fatome, "Polarization domain walls in optical fibres as topological bits for data transmission," Nature Photon. 11, 102 (2017).

[14] M. Haelterman, "Polarisation domain wall solitary waves for optical fibre transmission," Electron. Lett. 30, 1510 (1994); M. Haelterman and M. Badolo, "Dual-frequency wall solitary waves for nonreturn-to-zero signal transmission in W-type single-mode fibers," Opt. Lett. 20, 2285 (1995).

[15] J. Fatome, F. Leo, M. Guasoni, B. Kibler, M. Erkintalo, and S. Coen, "Polarization domain-wall cavity solitons in isotropic fiber ring resonators," in Nonlinear Photonics, NP'2016 (Optical Society of America, Sydney, NSW, Australia, 2016) p. NW3B.6.

[16] G.-L. Oppo, A. J. Scroggie, and W. J. Firth, "Characterization, dynamics and stabilization of diffractive domain walls and dark ring cavity solitons in parametric oscillators," Phys. Rev. E 63, 066209 (2001).

[17] D. V. Skryabin, A. Yulin, D. Michaelis, W. J. Firth, G.L. Oppo, U. Peschel, and F. Lederer, "Perturbation theory for domain walls in the parametric Ginzburg-Landau equation," Phys. Rev. E 64, 056618 (2001).

[18] I. Rabbiosi, A. J. Scroggie, and G.-L. Oppo, "Suppression of spatial chaos via noise-induced growth of arrays of spatial solitons," Phys. Rev. Lett. 89, 254102 (2002).

[19] S. Trillo, M. Haelterman, and A. Sheppard, "Stable topological spatial solitons in optical parametric oscillators," Opt. Lett. 22, 970 (1997); G.-L. Oppo, A. J. Scroggie, and W. J. Firth, "From domain walls to localized structures in degenerate optical parametric oscillators," J. Opt. B: Quantum Semiclass. Opt. 1, 133 (1999).

[20] V. B. Taranenko, K. Staliunas, and C. O. Weiss, "Pattern formation and localized structures in degenerate optical parametric mixing," Phys. Rev. Lett. 81, 2236 (1998); A. Esteban-Martín, V. B. Taranenko, J. García, G. J. de Valcárcel, and E. Roldán, "Controlled observation of a nonequilibrium Ising-Bloch transition in a nonlinear optical cavity," ibid. 94, 223903 (2005).

[21] Z. Wang, A. Marandi, K. Wen, R. L. Byer, and Y. Yamamoto, "Coherent Ising machine based on degenerate optical parametric oscillators," Phys. Rev. A 88, 063853 (2013); A. Marandi, Z. Wang, K. Takata, R. L. Byer, and Y. Yamamoto, "Network of time-multiplexed optical parametric oscillators as a coherent Ising machine," Nature Photon. 8, 937 (2014).

[22] T. Inagaki, Y. Haribara, K. Igarashi, T. Sonobe, S. Tamate, T. Honjo, A. Marandi, P. L. McMahon, T. Umeki, K. Enbutsu, O. Tadanaga, H. Takenouchi, K. Aihara, K.-i. Kawarabayashi, K. Inoue, S. Utsunomiya, and H. Takesue, "A coherent Ising machine for 2000-node optimization problems," Science 354, 603 (2016).

[23] Q. L. Williams, J. García-Ojalvo, and R. Roy, "Fast intracavity polarization dynamics of an erbium-doped fiber ring laser: Inclusion of stochastic effects," Phys. Rev. A 55, 2376 (1997).

[24] H. Zhang, D. Y. Tang, L. M. Zhao, and X. Wu, "Observation of polarization domain wall solitons in weakly birefringent cavity fiber lasers," Phys. Rev. B 80, 052302 (2009).

[25] C. Lecaplain, P. Grelu, and S. Wabnitz, "Polarizationdomain-wall complexes in fiber lasers," J. Opt. Soc. Am. B 30, 211 (2013).

[26] F. Marino, G. Giacomelli, and S. Barland, "Front Pinning and Localized States Analogues in Long-Delayed Bistable Systems," Phys. Rev. Lett. 112, 103901 (2014).

[27] L. A. Lugiato and R. Lefever, "Spatial dissipative structures in passive optical systems," Phys. Rev. Lett. 58, 2209 (1987).

[28] M. Haelterman, S. Trillo, and S. Wabnitz, "Polarization multistability and instability in a nonlinear dispersive ring cavity," J. Opt. Soc. Am. B 11, 446 (1994).

[29] E. Averlant, M. Tlidi, K. Panajotov, and L. Weicker, "Coexistence of cavity solitons with different polarization states and different power peaks in all-fiber resonators," Opt. Lett. 42, 2750 (2017).

[30] A. U. Nielsen, B. Garbin, S. Coen, S. G. Murdoch, and M. Erkintalo, "Coexistence and interactions between nonlinear states with different polarizations in a monochromatically driven passive Kerr resonator," Phys. Rev. Lett. 123, 013902 (2019).

[31] G. P. Agrawal, Nonlinear Fiber Optics, 5th ed. (Academic Press, 2013).

[32] A. E. Kaplan and P. Meystre, "Directionally asymmetrical bistability in a symmetrically pumped nonlinear ring interferometer," Opt. Commun. 40, 229 (1982); I. P. Areshev, T. A. Murina, N. N. Rosanov, and V. K. Subashiev, "Polarization and amplitude optical multistability in a nonlinear ring cavity," ibid. 47, 414 (1983).

[33] M. T. M. Woodley, J. M. Silver, L. Hill, F. Copie, L. Del Bino, S. Zhang, G.-L. Oppo, and P. Del'Haye, "Universal symmetry-breaking dynamics for the Kerr interaction of counterpropagating light in dielectric ring resonators," Phys. Rev. A 98, 053863 (2018).

[34] B. Garbin, J. Fatome, G.-L. Oppo, M. Erkintalo, S. G. Murdoch, and S. Coen, "Asymmetric balance in symmetry breaking," Phys. Rev. Research 2, 023244 (2020).

[35] F. Leo, S. Coen, P. Kockaert, S.-P. Gorza, Ph. Emplit, and M. Haelterman, "Temporal cavity solitons in onedimensional Kerr media as bits in an all-optical buffer," Nature Photon. 4, 471 (2010).

[36] A. J. Barlow, J. J. Ramskov-Hansen, and D. N. Payne, "Birefringence and polarization mode-dispersion in spun single-mode fibers," Appl. Opt. 20, 2962 (1981).

[37] M. Anderson, F. Leo, S. Coen, M. Erkintalo, and S. G. Murdoch, "Observations of spatiotemporal instabilities of temporal cavity solitons," Optica 3, 1071 (2016).

[38] S. Coen, M. Haelterman, Ph. Emplit, L. Delage, L. M. Simohamed, and F. Reynaud, "Experimental investigation of the dynamics of a stabilized nonlinear fiber ring resonator," J. Opt. Soc. Am. B 15, 2283 (1998).

[39] N. N. Rozanov, V. E. Semenov, and G. V. Khodova, "Transverse structure of a field in nonlinear bistable interferometers. I. Switching waves and steady-state profiles," Sov. J. Quantum. Electron. 12, 193 (1982), translated from Kvantovaya Elektron. (Moscow) 9, 354-360 (Feb. 1982); S. Coen, M. Tlidi, Ph. Emplit, and M. Haelterman, "Convection versus dispersion in optical bistability," Phys. Rev. Lett. 83, 2328 (1999).

[40] Y. Pomeau, "Front motion, metastability and subcritical bifurcations in hydrodynamics," Physica D 23, 3 (1986).

[41] F. Haudin, R. G. Elías, R. G. Rojas, U. Bortolozzo, 
M. G. Clerc, and S. Residori, "Front dynamics and pinning-depinning phenomenon in spatially periodic media," Phys. Rev. E 81, 056203 (2010).

[42] J. K. Jang, M. Erkintalo, S. Coen, and S. G. Murdoch, "Temporal tweezing of light through the trapping and manipulation of temporal cavity solitons," Nature Commun. 6, 7370 (2015); B. Garbin, J. Javaloyes, G. Tissoni, and S. Barland, "Interaction mediated trans- port of optical localized states in a trapping potential," arXiv:1710.01017 (2017).

[43] J. R. Pierce, "Coupling of modes of propagation," J. Appl. Phys. 25, 179 (1954).

[44] L. Del Bino, J. M. Silver, S. L. Stebbings, and P. Del'Haye, "Symmetry breaking of counter-propagating light in a nonlinear resonator," Sci. Rep. 7, 43142 (2017). 